\title{
Observations of Solar-Like Oscillations
}

\author{
Hans Kjeldsen \\ Teoretisk Astrofysik Center, Institut for Fysik og Astronomi, Aarhus \\ Universitet, DK-8000 Aarhus C, Denmark \\ Timothy R. Bedding \\ School of Physics A28, University of Sydney, NSW 2006, Australia
}

\begin{abstract}
The solar-like oscillations are oscillations that are expected to be excited by near-surface convection. Apart from the Sun itself, we now have a number of stars where p-mode solar-like oscillations have been detected. In this paper we present a short presentation of those recently discovered oscillations.
\end{abstract}

\section{Introduction}

The oscillations in the Sun are believed to be excited by convection near the solar surface, and we therefore expect that all stars that have a outer convection zone will display solar-like oscillations. A detailed discussion of solar-like oscillations, their detection and properties can be found in a recent review in Bedding \& Kjeldsen (2003), and we will therefore not discuss all of the details of solar-like oscillations in the present paper. In the description below we will concentrate on two aspects related to observations of solar-like oscillations; the global properties of the p-mode excess power and the oscillations detected in $\alpha$ Cen A.

\section{P-Mode Power in Solar-Type Stars}

Detailed model calculations of solar and solar-like oscillations as well as observations of high-order p-mode oscillations in a number of stars, show that the frequencies of low-degree $(\ell)$ and high-order $(n)$ oscillations can be approximated by the asymptotic relation:

$$
\nu_{n, \ell}=\Delta \nu\left(n+\frac{1}{2} \ell+\epsilon\right)-\ell(\ell+1) D_{0}
$$

This relation show that the structure of p-modes can be characterized by a set of so-called separations. The large separation $(\Delta \nu)$ is the distance between modes with same degree (but different order), while the small separations reflect differences between modes with different order (and different degree). The following small separations can be defined:

$$
\delta \nu_{02}=\nu_{n, \ell=0}-\nu_{n-1, \ell=2}=6 D_{0}
$$




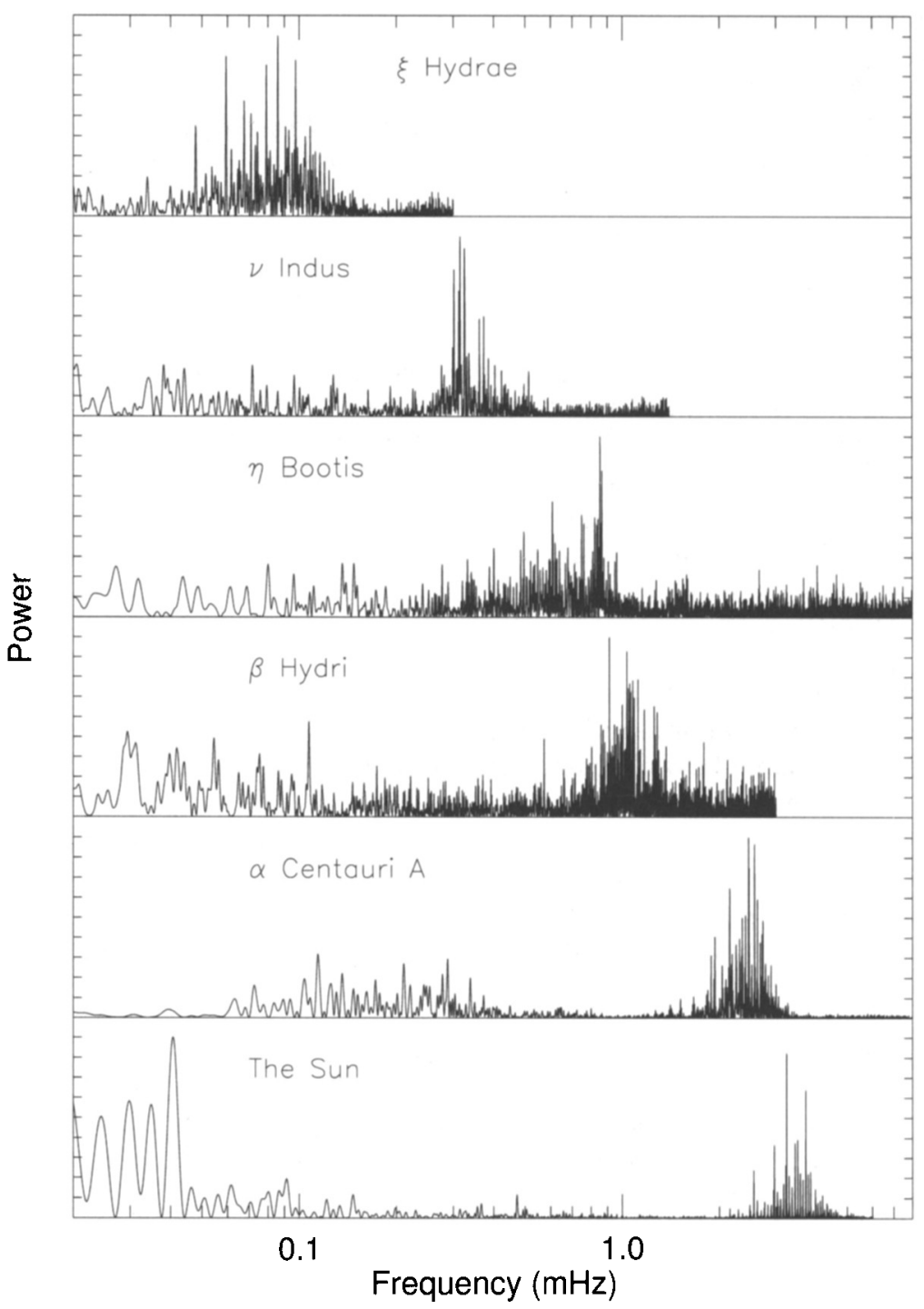

Figure 1. Observed power spectra of oscillations in the Sun and in 5 other stars. For details of the observations see Bedding \& Kjeldsen (2003). The frequency is shown on a logarithmic scale and, as one can see, oscillations are found at a significantly lower frequency in evolved stars like $\xi$ Hydrae, $\nu$ Indus, $\eta$ Bootis and $\beta$ Hydri than observed in $\alpha$ Cen $\mathrm{A}$ and the Sun. The reason for this is that the peak $\mathrm{p}$-mode power is proportional to the acoustic cutoff frequency in the stellar atmospheres. 


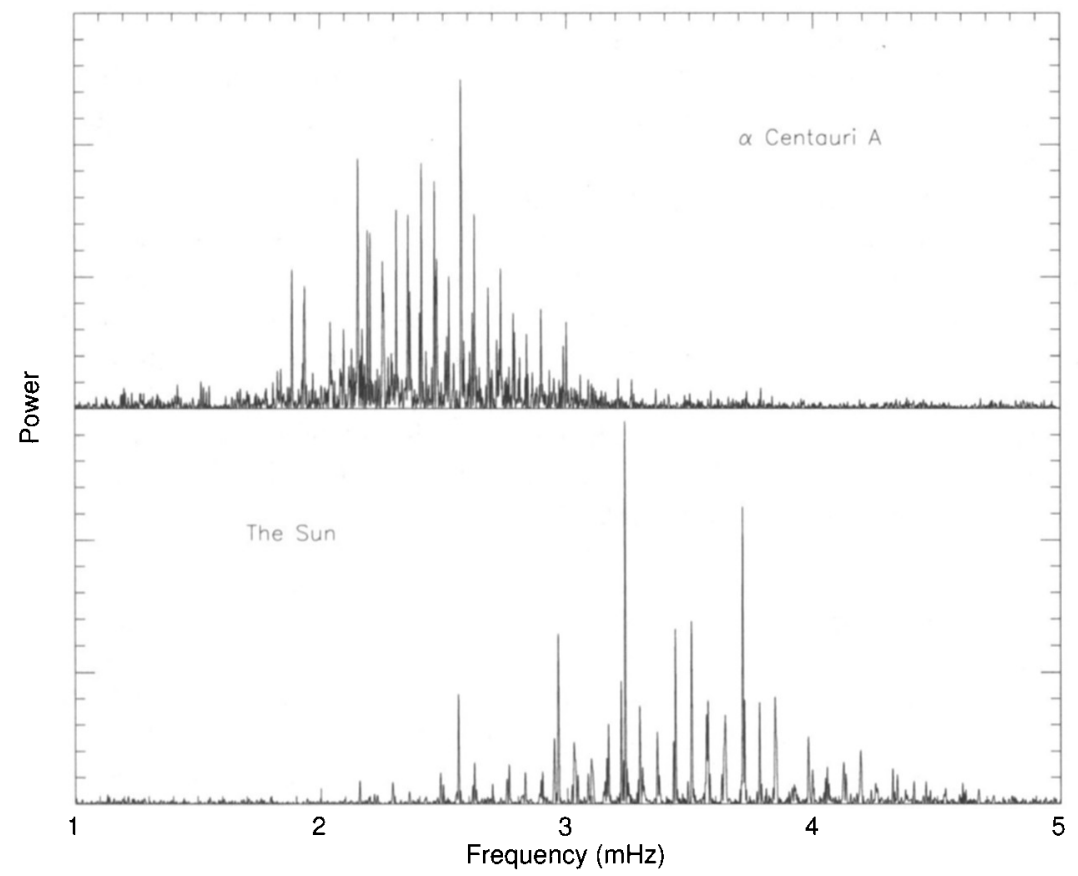

Figure 2. Power spectrum of time series of velocity measurements for the star $\alpha$ Centauri A and the Sun. The details of the power spectrum indicate that $\alpha$ Cen $A$ is significantly more evolved than the Sun. The large separation for the Sun is about $135 \mu \mathrm{Hz}$ and the small separation $\left(\delta \nu_{02}\right)$ is about 9 $\mu \mathrm{Hz}$. For $\alpha$ Cen $\mathrm{A}$ the same values are $106 \mu \mathrm{Hz}$ for the large separation and $5.5 \mu \mathrm{Hz}$ for the small separation $\left(\delta \nu_{02}\right)$. Based on the value for the large separation, we can estimate the density of $\alpha$ Cen A to be 0.61 times solar $\left(0.86 \mathrm{~g} / \mathrm{cm}^{3}\right)$. The $\alpha$ Cen A power spectrum in this figure is from Bedding et al. (2003) and the solar spectrum from full-disk velocities from the GOLF instrument on SoHO (Gabriel et al. 1997). The velocities for the $\alpha$ Centauri A time series show a scatter that is well below $1 \mathrm{~m} / \mathrm{s}$ per data point for data taken at the UVES spectrograph (using an iodine absorption cell for wavelength referencing) at VLT (UT2) at a sampling rate of two data points per minute. This accuracy is in fact higher than the one obtained using the GOLF instrument. The observations of $\alpha$ Cen A result in a noise level in the Fourier amplitude spectrum of $1.9 \mathrm{~cm} / \mathrm{s}$ and as such, these observations represent the most accurate velocities ever measured on any star (apart from our Sun). 


$$
\delta \nu_{13}=\nu_{n, \ell=1}-\nu_{n-1, \ell=3}=10 D_{0}
$$

The small separations reflect details of the structure of the star while the large separation to first order is a measure of the stellar density (see e.g. Kjeldsen \& Bedding 1995). The small separations will decrease during the Hydrogen core burning phase of the stellar evolution and one will therefore potentially be able to determine stellar ages by detailed measurements of the small separations (see e.g. Brown et al. (1994); Gough (2003); and Christensen-Dalsgaard in this volume).

Observing solar-like oscillations is difficult because of the low amplitude (peak amplitudes for individual modes are in most cases below $1 \mathrm{~m} / \mathrm{s}$ ). The frequency of low-degree high-order modes are in most cases between 0.5 and 4 $\mathrm{mHz}$ making observations insensitive to low-level night-to-night variations. The first star (apart from the Sun) where solar-like p-mode excess power was found is Procyon ( $\alpha \mathrm{CMi}$ ), an F5 subgiant. Evidence for oscillations was presented as early as 1991 by Brown et al. Since then detections have been claimed in a number of stars, some of them shown in figure 1. For details on the individual stars, see Bedding \& Kjeldsen (2003).

\section{Seismology of $\alpha$ Cen A}

The star $\alpha$ Cen A is a nearby star with the same spectral type as the Sun and it is therefore an obvious target for trying to detect solar-like oscillations. Using the CORALIE spectrograph, Bouchy \& Carrier (2001 and 2002) detected p-modes similar to those seen in the Sun. New observations using the UVES instrument (on VLT) and the UCLES spectrograph (on AAT) confirm the frequencies detected by Bouchy \& Carrier (see figure 2).

Acknowledgments. This work was supported in part by the Danish National Research Foundation through its establishment of the Theoretical Astrophysics Center.

\section{References}

Bedding, T. R., \& Kjeldsen, H. 2003, PASA, 20, 203

Bouchy, F. \& Carrier, F. 2001, A\&A, 374, L5

Bouchy, F., \& Carrier, F. 2002, A\&A, 390, 205

Brown, T. M., Gilliland, R. L., Noyes, R. W. \& Ramsey, L. W. 1991, ApJ, 368, 599

Brown, T. M., Christensen-Dalsgaard, J,, Weibel-Mihalas, B. \& Gilliland, R. L. 1994, ApJ, 427, 1013

Bedding, T. R., et al. 2003, Ap\&SS, 284, 303

Gabriel, A. H., et al. 1997, Solar Phys., 175, 207

Gough, D. O., 2003, Ap\&SS, 284, 165

Kjeldsen, H., \& Bedding, T.R. 1995, A\&A, 293, 87 